\title{
Cellular Interactions in the Development of the Shell Gland of the Gastropod, Ilyanassa
}

\author{
JAMES N. CATHER \\ Associate Professor of Zoology, The University of Michigan, \\ Ann Arbor, Michigan
}

\begin{abstract}
To determine the cell lineage of the shell gland in Ilyanassa, the $2 d$ and $2 c$ micromeres were marked with carbon granules. The $2 d$ micromere derivatives form the shell gland, but $2 c$ derivatives are later incorporated into the mantle. Deletion of $2 \mathrm{~d}$ causes shell abnormalities but, in about $10 \%$ of the cases, the shell appears normal although somewhat small. Deletion of $2 \mathrm{c}$ also causes characteristic shell abnormalities. After deletion of both $2 \mathrm{~d}$ and $2 \mathrm{c}$, no shell forms. When $2 \mathrm{c}$ is marked and $2 \mathrm{~d}$ deleted, the shell gland is marked. Any one-quarter embryo can form internal shell masses. In 1013 isolated ectoblasts ( $-3 \mathrm{~A},-3 \mathrm{~B},-3 \mathrm{C},-3 \mathrm{D})$, no shell material formed. Ectoblast with any single macromere formed shell with good morphogenesis, as did ectoblast plus $4 \mathrm{~d}$. Posterior ectoblast plus $4 \mathrm{~d}$, equivalent in size to isolated whole ectoblast, formed external shell. Properly oriented ectoblast, grown in contact with a polar lobe, formed shell in up to $70 \%$ of the cases; in one-half of these, the shell was external. Deletion of the macromere from an ectoblast plus $3 \mathrm{C}$ series of embryos at intervals through the first day resulted in embryos which did not form shell. Ectoblast in contact with polar lobe for six days, then separated, developed shell on the seventh or eighth day. (1) Neither ectoderm nor endoderm in isolation will form shell but any ectoderm-endoderm combination has the histogenetic ability to form shell material; (2) the endoderm exerts its influence between the second and sixth days of development; (3) only those combinations including or in contact with polar lobe cytoplasm through the third quartet stage undergo normal morphogenesis; (4) transplanted polar lobes can induce ectoderm to form shell; (5) the histogenetic ability to form shell is suppressed or unactivated when polar lobe cytoplasm is present, except in the D quadrant; (6) specialization of the $D$ quadrant is one of the major evolutionary trends in spiralians.
\end{abstract}

Evidence for correlative differentiation - the developmental modifications due to the interaction between cells and tissues - in embryos from spirally cleaving mosaic eggs has generally eluded investigators since the origin of the theory of mosaic development three-quarters of a century ago. Classical studies of embryos from eggs from which the polar lobe was removed (lobeless embryos) by Crampton (1896) and Wilson ('04a) and of isolated blastomeres by Wilson ('04b) and Costello ('45) indicate a progressive segregation of capacities during development, so that at each cleavage there is a part of the embryo formed with the potentiality to form a particular structure and a remainder which does not have such a potential. This, in itself, does not necessarily imply that the whole embryo had such a capacity prior to the segregative cleavage, as is often stated; and the evidence for such an idea is, at best, equivocal. Isolated blastomeres or groups of blastomeres tend to self-differentiate, so that each cell will form the same structure in isolation as it would in the intact embryo. Therefore the prospective potency of the isolated or transplanted blastomere is said to be the same as the fate of the blastomere in the embryo. The potency may or may not equal the fate but will not surpass it, according to the theory. Other evidence indicates that the differentiative capacities of the blastomeres are fixed through a precocious segregation of qualitatively different cytoplasmic areas, especially the cortex, by a highly determined cleavage pattern to form a mosaic of self-differentiating cells (Raven, '58).

Although self-differentiation is dominant during the early development of spiralians, great correlative capacities are shown in the regenerative ability of these forms after metamorphosis. At present, little is known of the transition of the embryo from a group of independent blastomeres, precisely arranged and tightly bound to one 
another, into a highly integrated organism capable of delicate responses to the developmental environment. Whether the capacity for correlation develops gradually or instantaneously is unknown, as is the temporal assumption of correlative abilities in the different structures or systems in the individual.

Although early development was demonstrated to be primarily due to self-differentiation, a large number of studies have indicated a special developmental significance for the $\mathrm{D}$ quadrant, in addition to the origin of the mesoderm. Clement's investigations ('52, '56, '62) are the most complete in this regard. He has shown that $\mathrm{D}$ quadrant material, in this case derived from the polar lobe, is necessary for the establishment of normal symmetry, as a regulator of the rate and pattern of cleavage in the D quadrant, and for the normal morphogenesis of the foot, shell, eyes, otocysts, and heart. The potency of the foot and shell does not exceed the fate but D quarter embryos can produce eyes which are normally derived from $1 \mathrm{a}$ and $1 \mathrm{c}$ (Clement, personal communication) so that the $D$ quadrant retains a significant ability to regulate. Thus its potency can exceed its fate. Furthermore, Clement has clearly demonstrated that the morphogenetic influence of the polar lobe region is exerted during the formation of the four quartets of micromeres but that not all lobe-dependent larval features are determined at the same time. External shell appears only after the formation of the second quartet; however, normal morphogenesis of the shell, as well as the foot, velum and eyes, occurs only after the formation of the third quartet. The heart and intestine are determined with the formation of $4 \mathrm{~d}$. The $4 \mathrm{D}$ macromere is evidently nutritive and of no morphogenetic value. Although cytoplasmic segregation appears adequate to account for the determination of the intestine and heart, it is necessary to look to a mechanism of inductive nature to explain the relation of the polar lobe to the morphogenesis of shell, foot, velum, and eyes. Clement's ('62) experiments indicate that the fate of all the cells in mosaic eggs is not irrevocably determined at some early stage but is progressively determined through development. Certainly the polar lobe or D quadrant cytoplasm can be considered the primary morphogenetic organizer of molluscan development.

Other evidence for regulative ability comes from the studies of Penners ('37), who showed that the mesentoblast cell (4d) of Tubifex, under suitable conditions, can form ectodermal structures normally formed from the primary somatoblast (2d), which derives pole plasm from the same area of the egg as the mesentoblast. Unfortunately, this work has never been confirmed or extended to other species. Novikoff (' $38 \mathrm{a}$ ) showed that twins could develop from a Sabellaria egg when the formation of the polar lobe was suppressed so that the first cleavage was equal. Rather than a true correlative interaction between cells, this can be interpreted as an equal distribution of lobe substance providing each of the two cells with its organizer. Carefully designed experiments (Novikoff, ' $38 \mathrm{~b}$ ) failed to demonstrate cellular or lobe interaction in Sabellaria. Costello ('45) showed that gastrulation would occur in Nereis only in those blastomere combinations which include both endoderm and ectoderm. Using combined animal and vegetal tiers or vertical half-combinations, Hörstadius ('37) failed to demonstrate cellular interactions in embryos of the nemertine, Cerebratulus. No comparable experiments have been done with mollusks.

Raven's series of studies of lithiumtreated Limnaea embryos provide two valuable cases in the analysis of regulative ability in mollusks (Raven, '58). The first involves the differentiation of head structures, particularly the eyes and tentacles. A gradient-field concept was developed in which there is a peak of activity at the animal pole, the region which later becomes the apical plate. The lateral cephalic plates, where the eyes and tentacles form, are areas of intermediate dominance. Raven explained the abnormalities produced by lithium - synopthalmia, cyclopia, acephaly - on the basis of gradient suppression. Although this concept has provoked valuable experimental analysis the work of Verdonk ('65) carried out in Raven's laboratory makes the concept questionable. Verdonk showed that lithium treatment modifies the direction of cleavage in specific cells. These cells, which cease division 
in the normal embryo, then continue to divide to form a cluster of small cells. The treatment, in this case, appears to release certain cells from a division inhibitor. However, other cells which normally continue division are blocked by such treatment so that their progeny are not formed. The end result is that the anlagen of the eyes and tentacles are displaced so that they give rise to the structure equal to their fate but in the wrong position. Modification of cleavage pattern alone can account for the abnormalities and regulation is not demonstrated. Although the cortex may represent a prepatterned series of anlagen sites, no gradient hypothesis need be invoked.

Raven's ('52) analysis of the development of the molluscan shell gland showed that its differentiation is dependent on tissue interaction. Raven found that lithium treatment at certain stages leads to a displacement of the archenteron. Normally, the shell gland forms from the ectoderm at the point where it contacts the archenteron. When the archenteron is displaced, the shell gland forms wherever contact with the ectoderm is made, whether pretrochally or post-trochally. In exogastrulae, where the endoderm fails to contact the ectoderm, no shell gland forms.

Hess ('56a, '57), while studying Bithynia and later Limnaea, found that a secondary invagination of the digestive gland endoderm can induce shell gland but that never more than one shell gland forms in any embryo. A number of investigators (Hess, '56a, '57; Morrill and Gottesman, '62; Clement, '62) have shown that shell can be formed from $\mathrm{AB}$ half-embryos, and Clement has shown that any quarter-embryo can form shell. Hess ('56b) also determined that more than one part of the endoderm has the capacity to induce the ectoderm to form shell and that this capacity is retained for some time, as judged from the response of the ectoderm to migration of endodermal fragments into the embryo in late exogastrulae. Ranzi ('31) found that even isolated fragments of the shell gland formed shell in the cephalopod, Loligo.

Collier ('64) has pointed out that the interaction between the archenteron and ectoderm cannot occur in Ilyanassa, which gastrulates by epibole. This is true of a large part of molluscan species where the shell gland forms prior to the formation of the endoderm in the proximity of the shell gland.

The present investigation was initiated to determine the role of induction in the formation of the shell gland in mollusks.

\section{METHODS}

The experiments were performed on the prosobranch gastropod Nassarius obsoletus Say, commonly known to embryologists by its subgeneric name Ilyanassa, which will be used hereafter. Animals were obtained from the Marine Biological Laboratory, Woods Hole or were collected on the coast of the Olympic Peninsula in Washington.

Operation and culture methods are given in detail by Clement ('62) and will only be summarized here. Eggs or embryos removed from a capsule were carefully washed in pasteurized sea water and divided into control and experimental groups. When eggs had reached the stage of development required, the cell which was to be deleted from the experimental embryo was punctured with a fine glass needle. The injured cell cytolysed within a few minutes and fell away from the operated embryo, which was then checked for other damage and transferred into fresh pasteurized sea water. The number of embryos from a capsule which could be subjected to the same operation depended on the complexity of the operation and the length of time that the embryo remained at a suitable stage. The experimental embryos were observed several times during the first day to make sure that no cells killed unintentionally were present.

The embryos were allowed to develop until a well-formed shell was present in the controls or until larval differentiation was completed and all of the visible yolk was utilized. Embryos were observed in living condition, then relaxed in chloretone, fixed in $70 \%$ ethanol, and preserved as whole mounts in diaphane (Clement and Cather, '57). All specimens were observed in polarized light to determine the extent of the highly birefringent aragonite of the shell. Embryos for cell lineage studies were prepared in a similar manner 
or according to the method of Clement ('52) or Cather ('58).

Marking experiments were done by two methods which are standard for the study of vertebrate embryos but which, to my knowledge, have not been previously applied to the much smaller invertebrate embryos. In the first method, a piece of agar, containing nile blue sulfate was embedded in the wax-side of a depression previously formed in an operating dish. An embryo in a drop of pasteurized sea water was then appropriately positioned in the depression so that the proper cell was in contact with the dyed agar. The cell remained in contact with the agar for 30 minutes to one hour after which the embryo was placed in pasteurized sea water and checked to determine that only one blastomere was stained. The development of the stained and unstained areas was observed for as long as they could be distinguished, at best for four or five days. By this itme the shell gland was functional. In the second method, carbon marking was done wtih a $12 \mu$ glass ball formed on the end of a thin glass rod. The ball was immersed in sea water, touched to carbon particles spread on a paper towel and then brought in contact with the appropriate blastomere. Most of the carbon was usually incorporated into the cell but often some would remain on the surface. In both cases, however, the carbon appeared to be limited only to the daughter blastomeres of the marked cell. In some cases, carbon could not be detected after fixation. It was probably either so dispersed as to be invisible or it became separated from the cell after the period of initial observation instead of being incorporated. Carbonmarked embryos were prepared as whole mounts or fixed in $2 \%$ buffered osmium tetroxide, embedded in Epon, and sectioned at two $\mu$ with a Porter-Blum microtome. The specimens were then stained with $0.1 \%$ azur B bromide in Clark-Lubbs buffer at $\mathrm{pH} 8.5$ or in $0.1 \%$ basic fuchsin in $50 \%$ acetone. Thin sections were observed with RCA electron microscopes. Other histochemical techniques used were those from Pearse ('61).

Operating dishes for polar lobe transplantation were made by melting dental wax and pouring a layer into a disposable plastic Petri dish. To extract toxic materials from the hardened wax, the dish was filled with glass distilled water overnight. The dishes were drained and filled with pasteurized sea water, after which depressions were made in the wax, which were larger in diameter and somewhat deeper than an isolated polar lobe. An isolated ectoblast was first placed in the depression, the polar lobe was placed over it and the two pushed into close contact.

\section{RESULTS}

\section{Origin and development of the shell gland}

\section{A. Normal Histogenesis and Morphogen- esis}

The development of the shell gland in Ilyanassa is typical of that described in other prosobranch gastropods (Raven, '58; Manigault, '60; Fretter and Graham, '62; Wilbur and Yonge, '64).

In Ilyanassa, five stages of shell gland development can be recognized. The time given for the initiation of each stage was measured from anaphase of Meiosis I, the earliest stage readily available (Cather, '63), for embryos reared at $20 \pm 1^{\circ} \mathrm{C}$. Variation in the initiation of stages by embryos within a single capsule increased with time. There was as much as five hours difference between the first embryo to go into stage three and the last one to do so, and as much as ten hours difference by stage five.

The ectodermal cells which eventually form the shell gland show no visible signs of differentiation until about 66 hours, when the embryo elongates anteriorly as the stomodeum is formed. However, the ectodermal area which forms the shell gland can be followed, using the posterior turret cell and the 4D macromere as natural markers.

Stage 1 - Plate formation. The ectoderm cells which form the shell gland near the mid-dorsal area of the posterior end of the embryo become cuboidal while the rest of the posterior ectoderm remains more or less squamous. The nuclei enlarge and nucleoli persist throughout interphase. There is a progressive development of basophilia during this stage which persists until stage five. This is evidently due to 
RNA synthesis, since it is lost after ribonuclease digestion (Pearse, '61). By 76 hours, the cells have become columnar and form a flat plate which extends along and to the left of the dorsal midline of the embryo's posterior end (fig. 1).

Stage 2 -Invagination. At about 80 hours, the center of the flattened plate begins to invaginate to form a small pocket which, for a time, is almost closed from the exterior. The surrounding cells form the typical rosette which makes the shell gland particularly obvious at this stage. A thin membrane is formed at the apical ends of the cells, where it remains attached for a time by microvilli before lifting from the cells to close the opening of the invagination. The membrane persists through the early development of the shell gland and shell is continuously formed and always attached at the edge of the shell gland or mantle. This becomes the periostracum of the larval shell. The invagination is completed by 90 hours (fig. 2) when a somewhat viscous material - relative to sea water - is present in the invagination.

The highly organized cytoplasm developed at this stage is shown semidiagrammatically in figure 3 . The mitochondria become polarized so that their long axes parallel that of the cell. Mitochondria, in this stage, are most abundant in the apical half of the cell whereas they were more or less evenly distributed earlier. A large vesicle appears basally to the nucleus in each cell and almost completely fills the area between the nucleus and cell membrane. The matrix of this vesicle is intensely stained by basic fuchsin and azure $\mathrm{B}$ bromide. Minute birefringent granules appear within the vesicular matrix near the end of the stage. These are only visible in living material but are evidently represented by small clear circles in fixed and sectioned material. The endoplasmic reticulum is well developed as cisternae and tubules in the medial part of the cell surrounding the nucleus. Rough vesicular elements persist throughout the cytoplasm. The Golgi apparatus is most often seen, in the early part of this stage, near the nucleus on the apical side. There is a loss of yolk granules and lipid during this period.

Stage 3-Evagination. Over the next ten or more hours the shell gland evaginates, first to form a cup-shaped structure, then flattening to become saucer-shaped, and finally becoming convex (fig. 4). Other than the development of a very strong alkaline phosphatase reaction near the end of the stage, no obvious changes in cellular components are apparent.

Stage 4-Shell formation. Deposition of calcium carbonate starts at about 120 hours. This occurs immediately before or during the first stage of torsion (fig. 5). The operculum also becomes birefringent at about the same time but there is no consistency in the order of appearance between the shell and operculum. The calcium carbonate in the larval shell consists entirely of aragonite (the crystalline structure was determined through x-ray diffraction by Dr. Donald Peacor, University of Michigan) and is highly birefringent. The thin membranous periostracum, formed at the time of invagination, becomes rather closely associated with the cells of the shell gland and there appear to be some fusions which persist as minute holes in the larval shell. The underlying viscous material evidently forms the matrix for crystal formation, which occurs beneath the periostracum. Only one morphological cell type can be distinguished in the shell gland and it evidently forms the membrane, secretes the viscous material and the calcium carbonate of the shell.

Stage 5-Mantle formation. After the secretion of the shell, the basal vesicles are no longer present in the central cells of the shell gland. As these cells lose their basophilia, they become very flat and show no morphological signs of activity. The marginal cells retain the characterisitcs of the stage 2-3 shell gland cells. From this time on, the term mantle is preferable since the initial phase of development is completed with the secretion of shell material.

The mantle edge appears to grow in the early stages through the incorporation of surrounding ectodermal cells, as well as through proliferation. Division figures seen in the shell gland never occur in cells which have major morphological criteria for differentiation present. The source of such dividing cells is unknown. 


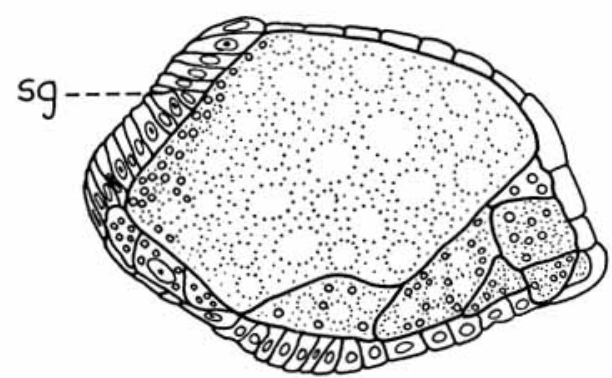

1

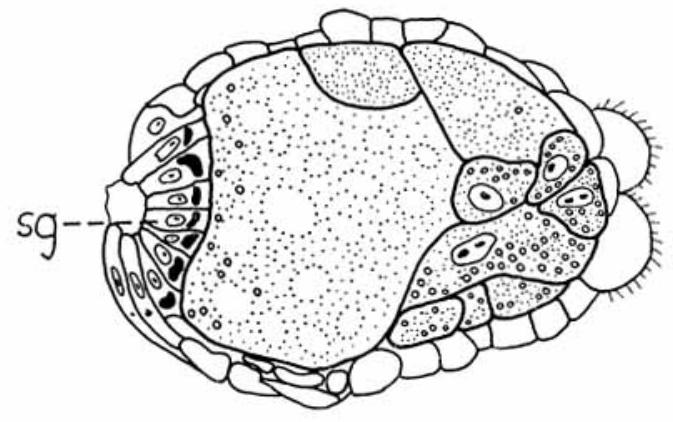

2

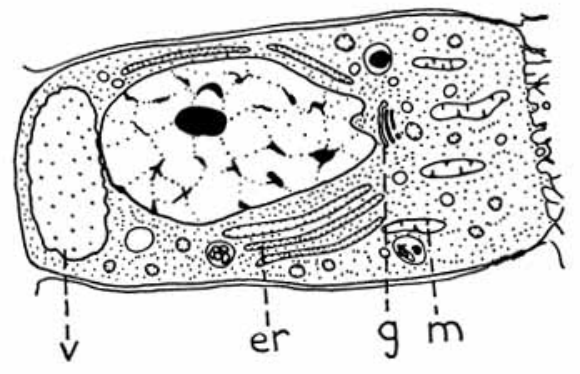

3
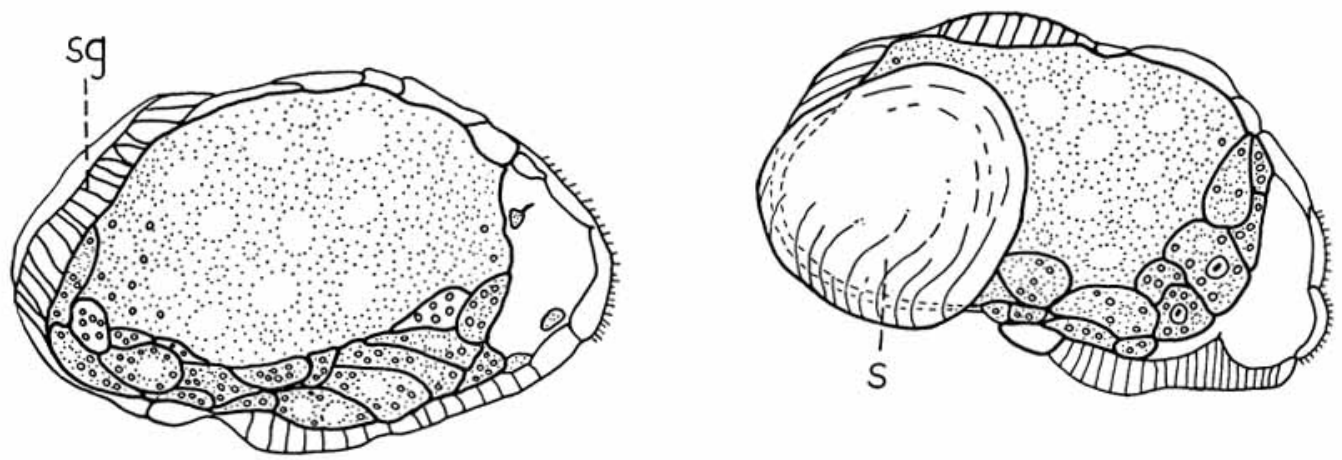

4

5

Fig. 1 Control embryo, 72 hours old. Shell gland stage 1, plate formation. Shell gland (sg).

Fig. 2 Control embryo, 90 hours old. Shell gland stage 2 , invagination.

Fig. 3 Shell gland cell from 96-hour-old control embryo near the end of stage 2. Endoplasmic re ticulum (er), Golgi complex ( $g$ ), Mitochondria (m), Vesicle (v).

Fig. 4 Control embryo, 108 hours old. Shell gland stage 3, evagination.

Fig. 5 Control embryo, 120 hours old, during early torsion. Shell gland stage 4 , shell formation ${ }^{\text {L. }}$ Shell (s). 
The veliger shell is almost bilaterally symmetrical. Its early growth is almost exclusively on the posterior and posteroventral side but the first phase of torsion rapidly shifts this small plate of shell to the right, so that the right side and posterior end are covered first by shell. Later the mantle edge grows forward dorsally and from the posterior along the left side to cover the visceral hump.

\section{B. Origin of the Shell Gland}

Lillie (1895) originally described the shell gland to be a derivative of the left posterior micromere of the second quartet (2d) in Unio, and Conklin (1897) agreed, although with some reservation, in his study of Crepidula. Later studies have all supported this lineage. However, my early operations in this series and the work of Clement ('56) and Hess ('56a) raised considerable doubt about the completeness of the early observations since shell was being formed from half and quarter embryos which lacked, not only the $2 \mathrm{~d}$ micromere, but in some cases the whole D quadrant. To determine the origin of the shell gland and mantle, the following experiments were done.

Cell marking experiments. Cells were marked with particles of carbon or stained with nile blue sulfate. In 21 embryos with the $2 \mathrm{~d}$ micromere carbon marked, the particles were found in the invaginating shell gland at 84 hours, as well as on the dorsal and ventral left side posterior to the larval kidneys, and along the dorsal and right posterior surface. The marked areas were not identical in every embryo but always included the area shown in figure 6. Many marked embryos were discarded because of questionable marking procedure or because of abnormalities. In six embryos which appeared to be normal, no carbon could be detected on the fourth day after marking.

In 12 embryos which had the right posterior micromere of the second quartet (2c) marked with carbon particles, the invaginated shell gland was unmarked but carbon was observed in the right lateral and ventral ectoderm. By 120 hours, a part of the marked area was being incorporated into the mantle edge (fig. 7), while the rest of this area appeared to form a part of the mantle cavity somewhat later. This was observed in four embryos which were fixed, after torsion had moved the intestine dorsally. In seven embryos which were marked, no carbon could be detected on the fourth or fifth day.

More than 100 embryos ( 87 of $2 \mathrm{~d}$ and 23 of 2c) were stained with nile blue sulfate. The marked areas on these embryos showed essentially the same pattern as the carbon-marked embryos of the same stages but the dye became progressively more diffuse, rendering these experiments less valuable than the carbon-particle experiments.

Second quartet micromere deletion experiments. The $2 \mathrm{~d}$ micromere was deleted from 100 embryos from 15 different capsules. Of these, 11 embryos formed shells which appeared to be perfectly normal, except that they were $75-85 \%$ the size of the shells of controls, on the ninth day of development (fig. 17). Thirty-two embryos formed cup-shaped shells, onehalf to one-third the size of the controls. Variable dumbbell-shaped shell plates were formed in 28 embryos (fig. 9). Internal shell masses were formed in 21 cases, all in the posterior half of the embryos. No shell formed in five cases.

Thirty-two embryos from six capsules in which the $2 \mathrm{c}$ micromere was deleted formed cup-shaped shells of one-half to one-third normal size. These were always severely flattened on the right side (fig. 19). The umbilicus was usually present. In contrast to the 2 d-deletion syndrome which is highly variable the 2c-deletion syndrome is extremely uniform. Deletion of $2 \mathrm{C}$ has no effect on shell formation (fig. 18).

In 27 embryos from six capsules in which the $2 \mathrm{c}$ and $2 \mathrm{~d}$ micromeres were both deleted, no shell was visible either internally or externally (fig. 8). The embryos appeared normal except for some shortening of the visceral hump. In three of the embryos, only one statocyst was present but, in all others, the foot was well formed and the statocysts and operculum were typically birefringent. Deletion of $2 a$, which is in a roughly comparable position to $2 c$ on the left side, has no effect on shell formation. 


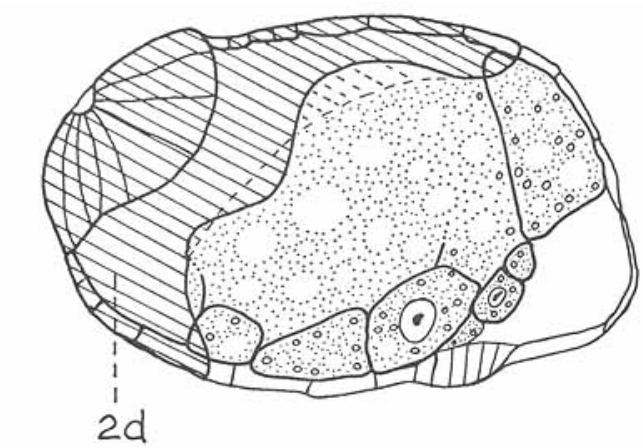

6

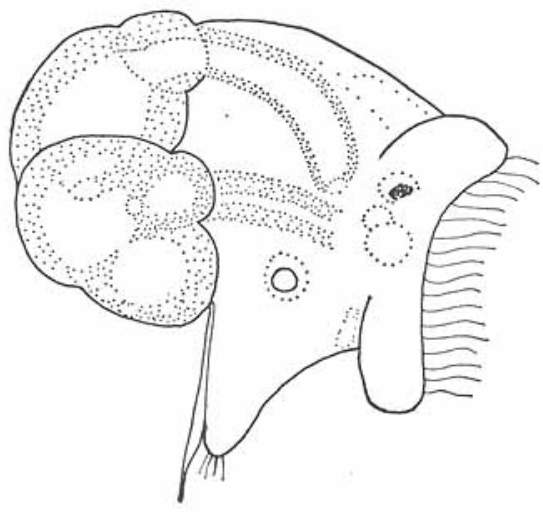

8

Fig. 6 Composite illustration where the hatched area represents the minimum distribution of carbon granules at 84 hours (stage 2) when the $2 \mathrm{~d}$ micromere was marked. Note the complete coverage of the shell gland at the upper left.

Fig. 7 Composite illustration where the hatched area represents the distribution of carbon granules at 120 hours (stage 4 ) when the $2 \mathrm{c}$ micromere was marked. Viewed from the upper right side. Shell (s).

Fig. 8 Larva, 168 hours old, from an egg lacking the $2 \mathrm{c}$ and $2 \mathrm{~d}$ micromeres. No shell is present.

Fig. 9 Larva, 168 hours old, from an egg lacking the $2 d$ micromere. The shell ( $s$ ) is represented by the hatched area.

Combination of cell marking and cell deletion experiments. In six embryos from six capsules, the $2 \mathrm{~d}$ micromere was deleted and the $2 \mathrm{c}$ micromere was marked with carbon particles. In five cases, the shell gland was considerably displaced to the right and appeared to be lagging behind the controls so that the marked area was comparable to an 84-hour shell gland in the controls, when the experimental embryo was 96 hours old. Since no additional delay was apparent after the formation of the shell gland, the rate of development thereafter was normal. One embryo was grossly monstrous and was not analyzed.

\section{Cellular interaction in shell gland development}

A. The Development of Isolated Ectoblasts

Since all of the ectoderm of gastropods comes from the first, second, and third quartets of micromeres, an embryo with- 
out mesoderm or endoderm - an ectoblast embryo - can be prepared by deletion of the $3 \mathrm{~A}, 3 \mathrm{~B}, 3 \mathrm{C}$, and $3 \mathrm{D}$ macromeres after the third quartet is formed. One thousand and thirteen operations of this type have been done and the resulting embryos have been studied for as long as 14 days of development.

The ectoblast embryo forms a hollow ball-like structure within 24 hours of the operation. Over the next 2-4 days, it becomes ciliated along a circumferential ring, near the more or less flattened base of the embryo. On the more pointed end, an apical tuft of cilia develops, associated with large cells which are probably apical plate cells. In some embryos, distinct areas lateral to the apical plate appear to be cephalic plates (figs. 10, 20). However, these areas cannot always be distinguished, possibly due to the difficulty in orienting the embryos.

Although these embryos live and are motile for at least ten days, no further differentiation of larval structures has been observed. In no case was any pigment, birefringent material, or other structural entity observed.

\section{B. The Development of Isolated Macro- meres}

A series of experiments were done in which quarter embryos were isolated. When the micromeres formed, they were deleted, so that isolated $3 \mathrm{~A}, 3 \mathrm{~B}, 3 \mathrm{C}$, and $3 \mathrm{D}$ macromeres were obtained. The first three macromeres are entirely endodermal, while the 3D macromere forms the mesentoblast cell ( $4 \mathrm{~d}$ ) as well as the $4 \mathrm{D}$ macromere. Twelve partial embryos of each type were isolated.

The endodermal blastomeres ( $3 \mathrm{~A}, 3 \mathrm{~B}$, 3C) all developed similarly, undergoing a few divisions, then becoming inactive and degenerating after a few days. The 3D macromere formed the mesentoblast cell which then divided a number of times. The 4D macromere was never observed to develop further. The mesentoblast derivatives often would adhere to the bottom of the dish and formed spindle shaped cells which persisted for from 10-15 days. Endodermal cells also frequently sent out pseudopodia and stuck to the substrate before their degeneration.
Several hundred macromeres, unidentified as to quadrant, were isolated in calcium-free artificial sea water but these developed so poorly and were so grossly abnormal that they were considered to be of no value.

No obvious larval structures developed in any case but the development of such cells in isolation is poor at best. The mesentoblast derivatives formed no structures but were never observed as a compact mass so their real capacity in isolation is unknown.

\section{Shell Development in Ectoblast-ento- blast Combinations}

Half- and quarter-embryos. Since calcium carbonate was dissolved by the method of fixation used by Clement ('56), he described shell only in $C D$ and $D$ isolates. However, he has more recently reported ('62) that A, B, C, or AB can form internal shell masses.

In 40-50 embryos of each type ( $A, B, C$, and $\mathrm{AB}$ ) more than $50 \%$ developed internal shell masses. In about $10 \%$ of the cases, more than one mass was present, with several containing three or more. Histological examination indicates that these masses are formed by typical shell gland cells arranged in closed sacs of various forms (fig. 15). The cells undergo the same sequence of changes as those in the normal shell gland so that, when shell is present, the surrounding cells are flattened with little basophilia.

In 40 cases of $\mathrm{AD}$ half-embryos, $33 \mathrm{de}$ veloped external shells, most of which were small and cap-shaped; seven of these had better morphogenesis characterized by the presence of an umbilicus. Four embryos formed internal shell masses, while three had no apparent shell. In 53 CD halfembryos, 37 showed external shells which were about a third larger than those of the AD half-embryos. Morphogenesis appeared essentially normal in 11 of these, although the shells were relatively smaller than the controls. Four embryos developed internal shell masses; the remainder developed no shell and were often quite abnormal in general development.

In $12 \mathrm{D}$ quarter-embryos, one developed an external shell tag, eight had internal shell masses, and three had no shell ma- 
terial visible. Development of $\mathrm{D}$ quarterembryos was poor with only 12 out of 60 experimental embryos growing to a stage roughly comparable to the veliger.

Ectoblast plus one macromere embryos. In one of the early attempts to remove the macromeres to make an ectoblast embryo, an anucleate yolk tag ( 12 by $16 \mu$ ) remained attached to the ectoblast. The resulting embryo formed a small cap of shell (fig. 21).

This result led to a systematic series of experiments in which three of the four macromeres were removed. In 12 ectoblast plus 3A embryos, all formed shell which, in four cases, was external, with an umbilicus and characteristic form. Of 12 ectoblast plus 3B embryos, one had no shell, six had internal shells, and five developed external shells, three of which showed good morphogenesis. In 12 ectoblast plus 3C embryos, nine developed external well formed shells, each with some evidence of an umbilicus (fig. 14); three had internal shell masses. In 26 ectoblast plus 3D embryos, two formed internal shell masses, while 24 formed external shells with good morphogenesis. These latter embryos had consistently well developed endodermal structures, as well as the heart and intestine which were absent or poorly developed in the other types. All ectoblast plus macromere embryos showed good morphogenesis of foot, velum, and eyes.

One series of experiments was done in which the $3 \mathrm{~A}, 3 \mathrm{~B}$, and $3 \mathrm{D}$ macromere were deleted at the third quartet stage and then, at intervals of $4,8,12$, and 16 hours after the stage at which the mesentoblast forms in the controls, the $3 \mathrm{C}$ macromere was deleted (four cases at each time). These experiments were done to determine the time of interaction between the ectoderm and endoderm. All embryos in the series developed as typical ectoblast embryos and lacked shell, foot, and pigmentation.

Ectoblast plus mesentoblast embryos. In 42 embryos from 11 capsules, all of the macromeres were removed about $40 \mathrm{~min}$ utes after the formation of the mesentoblast cell. These embryos formed no shell but did show a light pigmentation. Clusters of small yolk granules which were presumably from the mesentoblast were present in the internal cells of the embryo.
Clement (personal communication) noted that, in a case of ectoblast plus $4 \mathrm{~d}$, a shell, operculum, and eyes developed. The embryo had fair velar development. When this experiment was repeated, by deleting the $4 \mathrm{D}$ macromere about 40 minutes after mesentoblast formation and then waiting for an hour before deleting $3 \mathrm{~A}, 3 \mathrm{~B}$, and $3 \mathrm{C}$, I was able to confirm and extend his result. In 32 cases from five capsules, four embryos lacked shell, nine had an internal shell mass, and 19 an external shell (figs. 11,23) and foot, an intestine, heart, and some large muscles. Velar development was variable and either one or two eyes were present. In the best cases, these embryos were small but at least superficially normal, although the endoderm was too compact to be adequately analyzed.

Posterior one-half ectoblast plus mesentoblast embryos. In 12 embryos from seven capsules, the $\mathrm{AB}$ cell was killed at the 2-cell stage; the CD half was allowed to develop until an hour or more after the mesentoblast cell formed, at which time $3 \mathrm{C}$ and $4 \mathrm{D}$ were deleted. The embryo from such an operation therefore included the posterior half of the ectoblast and the mesentoblast cell, and was equal in size or smaller than an isolated whole ectoblast. All such embryos formed shell; in three cases it was internal but, in nine, an external shell and foot developed. The velum was variable and reduced and only one embryo had one eye. Pigment of the intestine was visible but the rest of the endoderm

Fig. 10 Larva, 168 hours old, from an egg lacking the $3 \mathrm{~A}, 3 \mathrm{~B}, 3 \mathrm{C}$ and $3 \mathrm{D}$ macromeres (an ectoblast embryo). Note the pattern of cilia and the absence of differentiated structures.

Fig. 11 Larva, 168 hours old, from an egg lacking $3 \mathrm{~A}, 3 \mathrm{~B}, 3 \mathrm{C}$ and $4 \mathrm{D}$ (an ectoblast plus mesentoblast embryo). The area of the shell ( $s$ ) is hatched. Note the eyes and velar cilia.

Fig. 12 Larva, 168 hours old, lacking $\mathrm{AB}, 3 \mathrm{C}$ and $4 D$ (a posterior ectoblast plus mesentoblast embryo). Note the large cells underlying the shell (s) in this case.

Fig. 13 Larva from an ectoblast and polar lobe combination where the ectoblast cells have completely overgrown the yolky polar lobe. Note the internal shell $(\mathrm{s})$ masses.

Fig. 14 Larva from an ectoblast plus $3 \mathrm{C}$ macromere combination. Note the form of the shell, eyes, and velum as well as the absence of the intestine.

Fig. 15 Section of an AB half-embryo ( $-\mathrm{CD}$ ) showing two internal shell ( $\mathrm{s}$ ) masses and the typical shell gland cells surrounding them. 

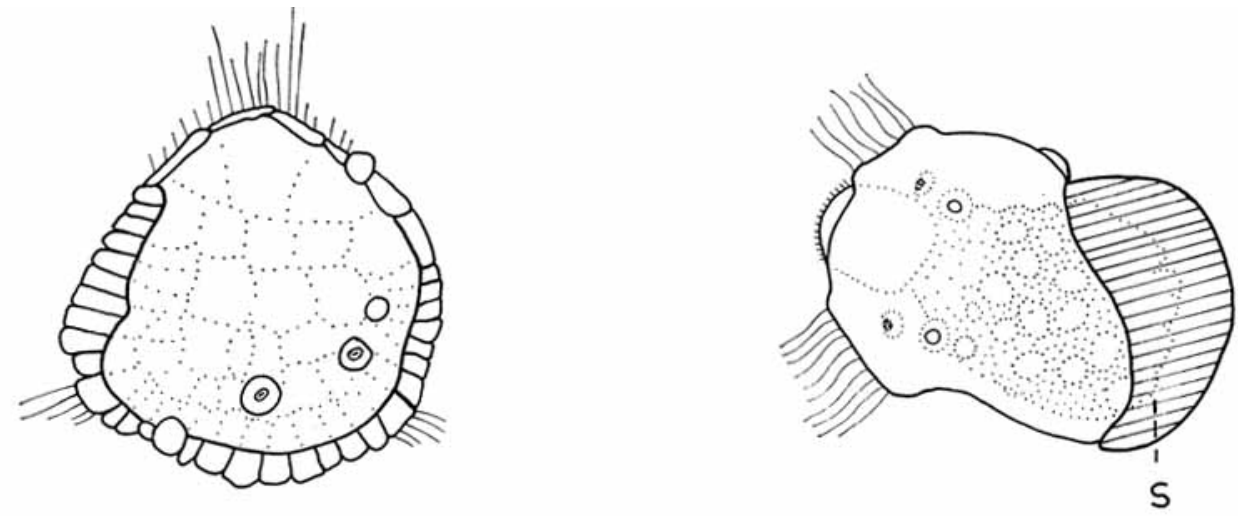

10

11
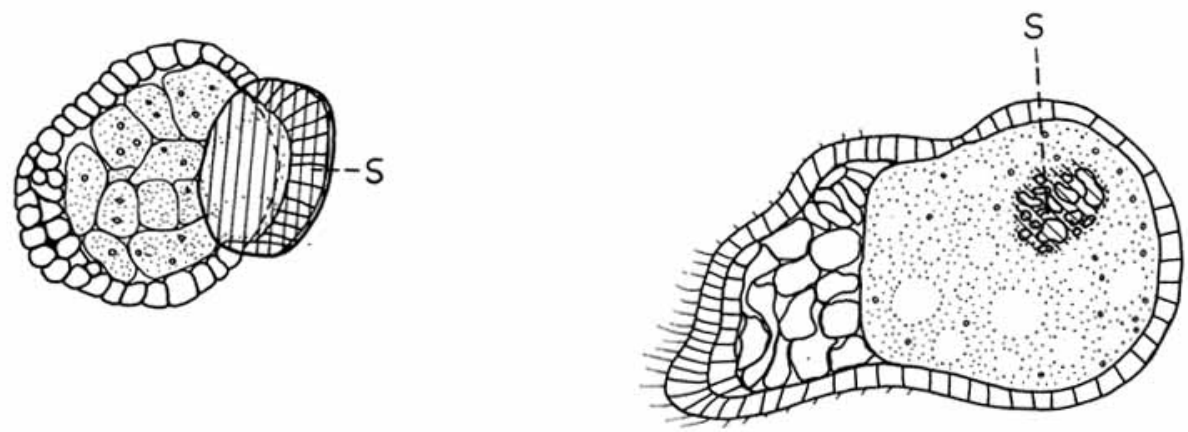

12

13
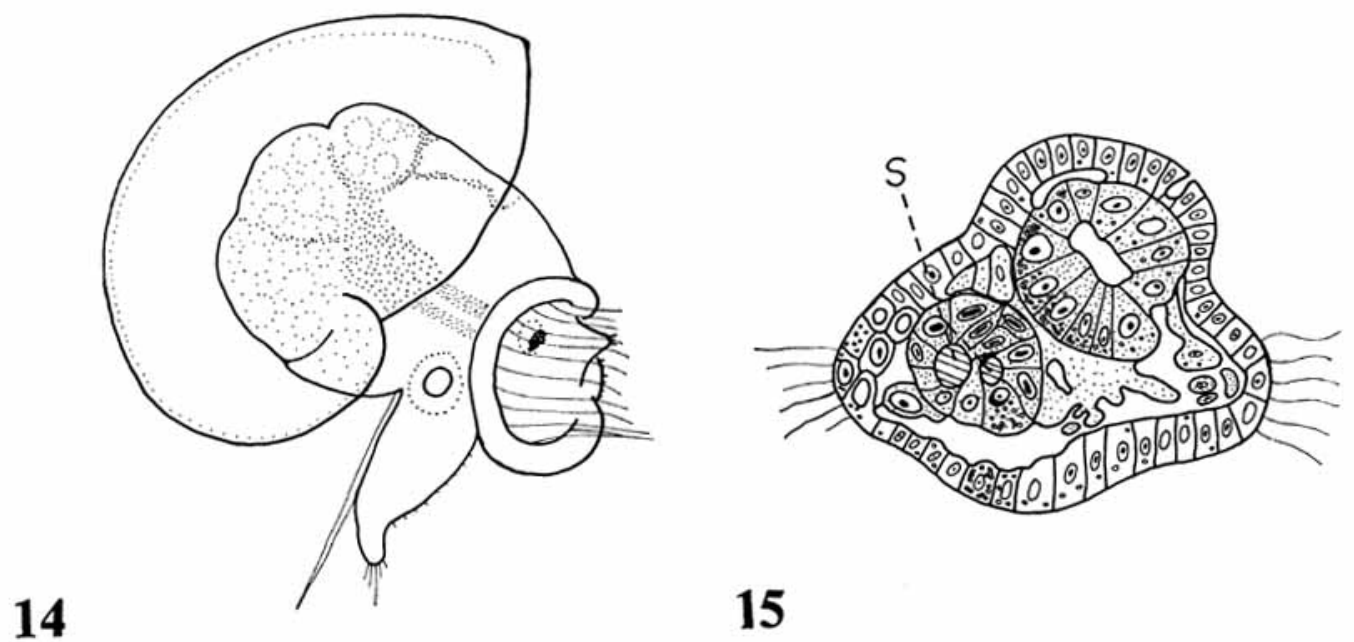

15

Figure 10-15 

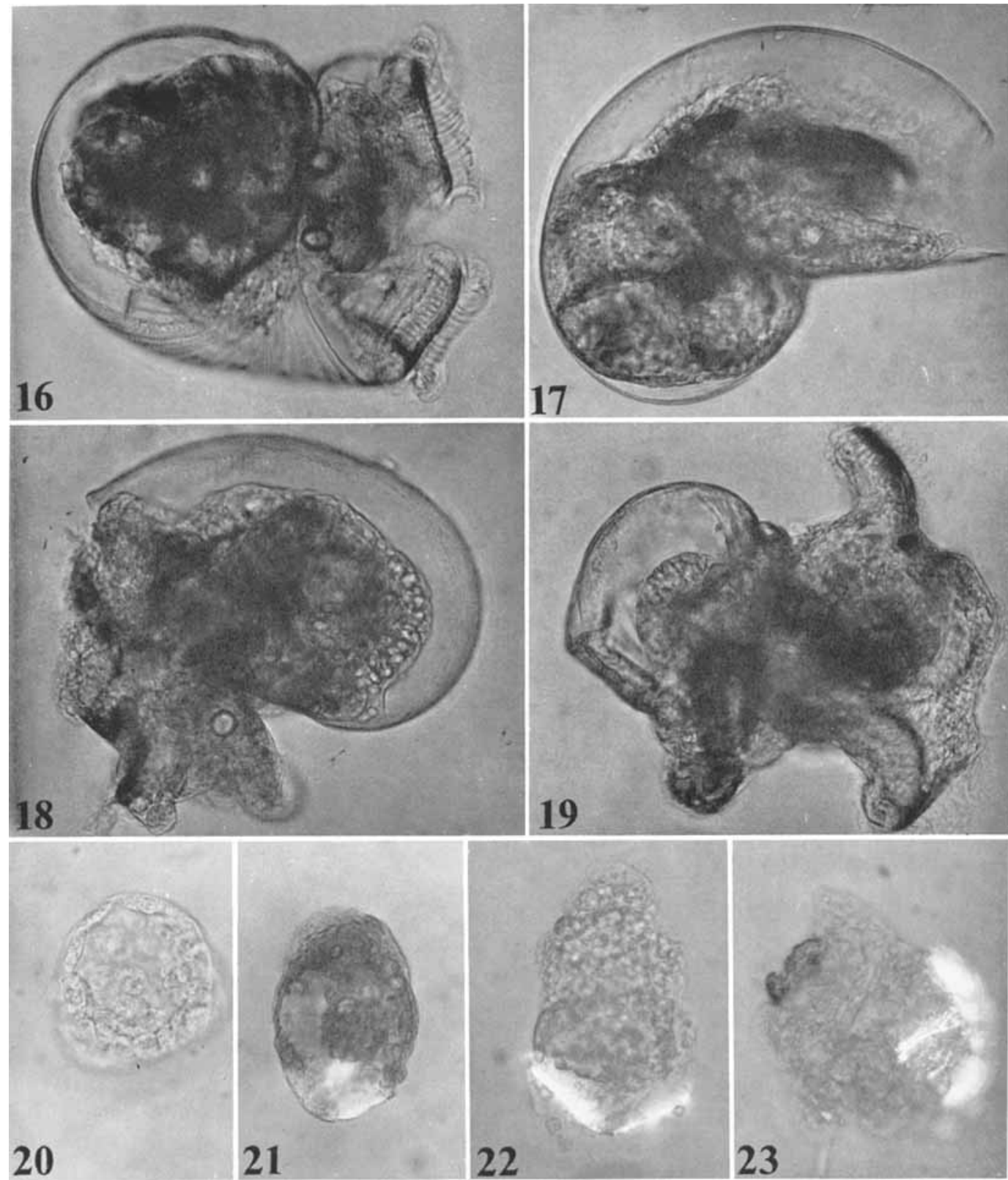

Fig. 16 Control veliger larva, 192 hours old. Ventral view. Note the pattern of pores in the shell in the lower part of the picture.

Fig. 17 Larva, 184 hours old, from an egg lacking the $2 \mathrm{~d}$ micromere. The animal is partly retracted into the shell.

Fig. 18 Larva, 168 hours old, from an egg lacking the $2 \mathrm{C}$ macromere. Note the form of the shell.

Fig. 19 Larva, 168 hours old, from an egg lacking the $2 \mathrm{c}$ micromere. Note the characteristic flattening on the right side of the reduced shell,

Fig. 20 Ectoblast larva (-3A, 3B, 3C, 3D), 144 hours old. Note the absence of differentiated structures.

Fig. 21 Embryo formed from an ectoblast plus a tag of yolky cytoplasm from an undetermined macromere. Photographed in polarized light to show the highly birefringent shell seen in the lower part of the picture.

Fig. 22 Eight-day-old embryo, separated from the polar lobe after six days of contact. Photographed in polarized light to show the shell.

Fig. 23 Ectoblast plus mesentoblast larva showing the shell, operculum, velar pigment and eye. Photographed in polarized light. 
and mesoderm was crowded and difficult to analyze (fig. 12).

Ectoblast plus polar lobe embryos. To combine ectoblasts with polar lobes the method was modified in several ways which affected the results. In all, however, ectoblasts were obtained as previously described and then eggs in the trefoil stage from another capsule were delobed with a fine glass hair. The ectoblast and the anucleate polar lobe were then placed in a suitably sized depression in a wax operating dish. In the first series of 100 embryos from 18 capsules left in the depression for seven days, six formed shell material of which four had internal masses (fig. 13) and two had small external pieces. Long cilia similar to velar cilia developed on the end opposite the shell in three of the elongate embryos. No other differentiated structures could be detected. Only in those cases where the ectoblast fused to the polar lobe so that the ectoblasts grew down over it, was shell formed. Only those ectoblasts placed so that the surface next to the original macromeres was in contact with the polar lobe fused with it and only those placed together within about two hours after the macromere deletion fused.

In a second series of 15 embryos from eight capsules, which were oriented so that the polar lobe was placed in contact with the ventral surface of the ectoblast and in which the duration of the operation was kept as short as possible, five developed shell. One had a cluster of internal shell granules, one formed an internal mass, and three developed small posterior shell plates. All were ciliated, but velar-like cilia were not observed.

In a third series, the polar lobes were removed in calcium-free artificial sea water and then placed with the ectoblasts. They were left in contact for six days and then removed from the depression. No fusion had occurred, but six of the $13 \mathrm{had}$ shell which was internal in three and external in three. Three of the ectoblasts which lacked shell on the sixth day, when they were separated from the lobe, developed shell by the eighth day (fig. 22). One embryo had internal granules and two had small shell plates which curved around the posterior end of the embryo. Some long cilia were present but these did not appear as large as velar cilia. One embryo had an internal cavity surrounded by columnar cells.

\section{DISCUSSION}

In order to evaluate critically the results of deletion and transplantation experiments done on the embryos of animals with mosaic development, the normal fate of the cells under consideration must be known. Otherwise it is not possible to distinguish between the self-differentiation of blastomeres and correlated interactions between them. All of the classic cell lineage studies derive the shell gland from the $2 \mathrm{~d}$ micromere (Raven, '58). It is not known which of the derivatives of $2 \mathrm{~d}$ actually forms the shell gland but $2 \mathrm{~d}^{11}$ forms part of the head vesicle in animals as widely separated phylogenetically as Limnaea (Verdonk, '65) and Crepidula (Conklin, 1897) and probably does also in Ilyanassa, although there is no specific information to support this point. Other structures ranging from the stomodeum to anal cells and foot have also been attributed to 2d, but these will require confirmation before they can be generally accepted. From Clement's ('58) study of the development of the foot, we now know that it originates from the third quartet micromeres, $3 \mathrm{c}$ and $3 \mathrm{~d}$, in Ilyanassa. Usually, in gastropods, the primary somatoblast (2d) is not as large as in lamellibranchs and annelids and it may not have as extensive a role in the formation of the posterior ectoderm.

The experimental evidence derived from the marking and deletion experiments presented here strongly supports the cell lineage studies on the origin of the shell gland. The participation of $2 \mathrm{c}$ in the formation of the shell and mantle cavity has not been previously described. In neither case does $2 \mathrm{c}$ form all of the structure. In the shell, $2 \mathrm{~d}$ is the major participant while in the case of the mantle cavity the left side remains unmarked after $2 \mathrm{c}$ has been marked. Although this could be artifactual, it may also indicate the participation of another blastomere, possibly $2 \mathrm{~d}$, but this could not be determined in the preparations made so far. Torsional movements make the development of the mantle cavity difficult to follow at the cellular level. 


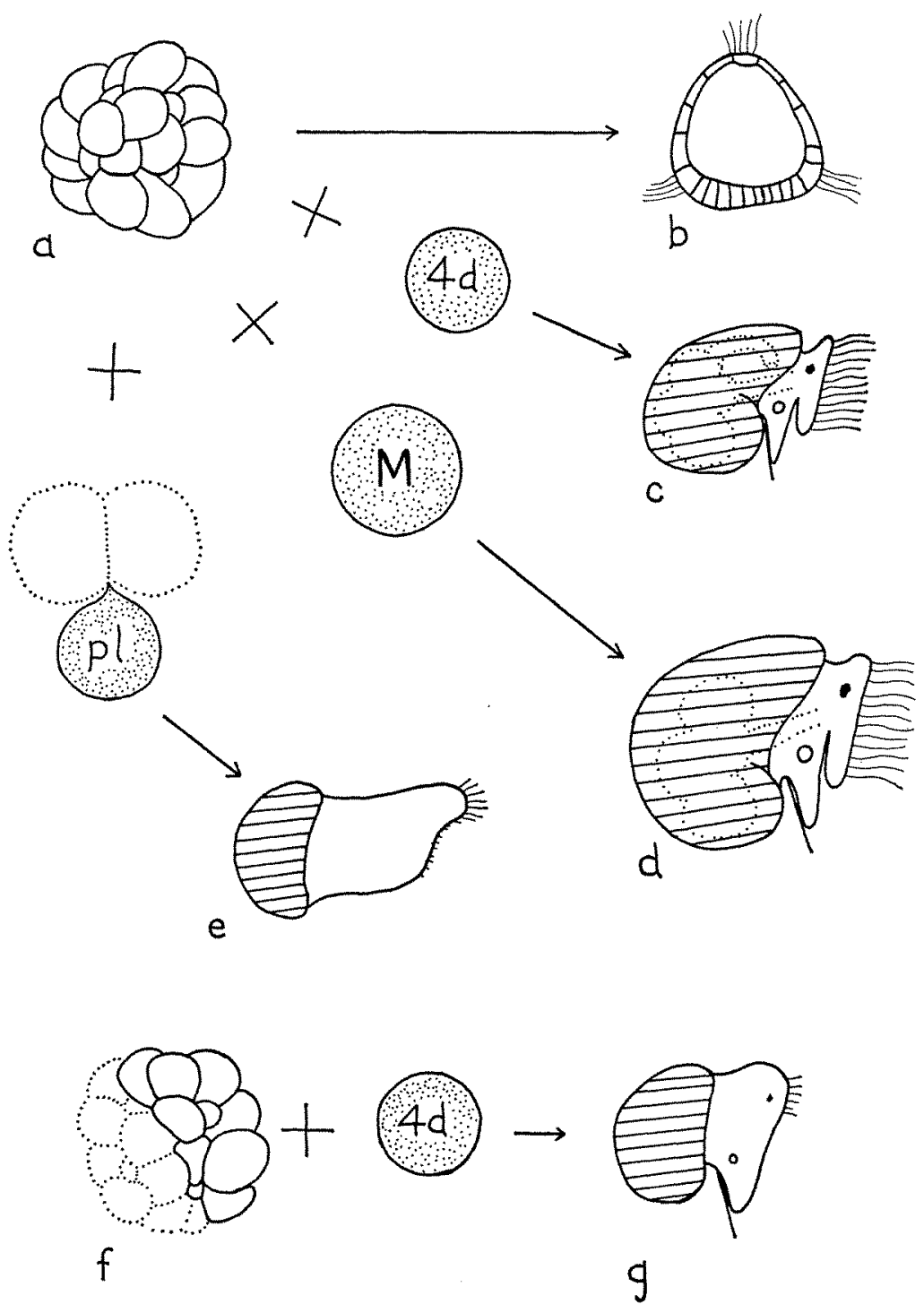

\section{4}

Fig. 24 A diagrammatic summary of the interactions preceding shell formation. Isolated ectoblast, (a). Larva from an isolated ectoblast, (b). Larva developed from an ectoblast plus mesentoblast (4d) combination in which the shell is hatched, (c). Larva developed from an ectoblast and macromere (M), (d). Larva developed from an ectoblast plus polar lobe (pl) combination. Dotted lines show cells which have been deleted, (e). Isolated posterior ectoblast, (f). Larva from a posterior ectoblast and mesentoblast cell combination, $(\mathrm{g})$.

The later development of the shell gland is typical of that previously described in other species, but it should be noted that the development of more than one type of differentiated cell does not occur in the shell gland of Ilyanassa, as it does in the mantle of many adult mollusks, nor are separate prismatic and nacreous layers formed in the larval shell. The actual formation of shell material is extracellular 
and occurs in the viscous material between the periostracum and the shell gland.

\section{Specificity and histogenesis}

Combinations of ectoderm and endoderm from any half- or quarter-embryo are capable of shell histogenesis. The present studies, however, indicate that neither ectoblasts nor endoblasts give rise to cells which will form shell material when isolated from the other type. Raven ('52) originally proposed that only small-celled endoderm could serve to induce the shell gland. Since the small-celled endoderm originates in each quarter in most mollusks, the apparent diversity of origin of shell material does not disprove his contention. Hess ('62) believes there is no great specificity of endoderm required, since even the digestive gland diverticulum formed in exogastrulae can serve as the inducer. Unfortunately, the origin of the digestive gland is not well enough known for one to critically evaluate this evidence, although it appears to indicate that the large-celled endoderm is also capable of inductive interaction. An attempt has been made to clarify this problem and to determine the inductive capacity of the endoderm and the competence of the ectoderm. Since any quarter-embryo can form shell material, both anterior and posterior and right and left ectodermal areas are competent to undergo the histogenetic differentiation necessary for shell secretion. External shell, however, has never been observed in Ilyanassa embryos which lack posterior ectoderm, but this appears to be related to the factors controlling morphogenesis rather than histogenesis. There does not appear to be great specificity of the posterior ectoderm derived from $2 d$ and $2 c$, since either can form the shell gland as well as other structures. The evidence indicates that specificity is established through correlated interaction with the endoderm, and possibly with the mesoderm, in the formation of mantle cavity structures. Raven ('52) showed that there is a considerable inherent cytodifferentiative ability in cells in exogastrulae even when morphogenesis does not occur, and when there is no close apposition of layers which might interact. He pointed out, however, that inherent histogenesis cannot account for shell gland formation in Limnaea as it cannot in Ilyanassa.

Clement ('62) and Collier ('64) have already noted that it is highly unlikely that the small-celled endoderm plays any role in the development of the shell gland in those animals with epibolic gastrulation such as Ilyanassa, because of the lack of proximity of the layers prior to the formation of the shell gland. In Ilyanassa, there is never any small-celled endoderm in the vicinity of the shell gland. The $4 \mathrm{D}$ macromere, which is in continuous contact with the derivatives of $2 \mathrm{~d}$, probably does not divide, at least not in the region of the shell gland, and the isolated polar lobe does not undergo divisions, yet both are capable of inductive interaction.

A possible interaction between the mesentoblast (4d) and the somatoblast (2d), during late cleavage when these cells are in close contact, must also be considered. Since embryos resulting from properly executed operations to form combinations of ectoblast and mesentoblast almost always form shell, this is a distinct possibility. In those cases where shell was not formed, there was probably a displacement of the mesentoblast which caused too great a separation for interaction to occur. The sequential macromere deletion experiment, however, indicates that the endoderm must be present for more than 24 hours of development for the interaction to occur. During this period in normal development, the mesentoblast derivatives are shifted away from the site of the shell gland so the mesentoblast is probably not the normal inducer.

As Collier ('64) has pointed out, the shell gland develops in ectoderm which has contact only with the 4D macromere. Contrary to his conclusion that induction is not probable, it appears that the 4D macromere is the inductive cell.

Costello ("45) has pointed out that the failure of differentiation of certain structures in small isolates and production of these structures by larger combinations of blastomeres is not, in itself, proof of induction. There are many cases known where a minimal cellular mass is required for differentiation.

When the posterior ectoblast is isolated with the mesentoblast, the mass is less 
than the whole isolated ectoblast yet the resulting embryo almost always secretes a small external shell. The cytoplasmic particles of the mesentoblast, such as fine yolk granules and lipid droplets distinguish it from the ectodermal cells. It appears that qualitative differences, whatever their bases may be, rather than quantitative ones determine the capacity to interact.

Thus it appears that any endoderm can serve to induce the ectoderm to carry out the necessary histogenetic changes for shell secretion within wide limits of size and relative proportions.

\section{Morphogenesis}

If the endoderm has such broad inductive capacity and the ectoderm such wide competence, how is it that shell does not form in each quadrant of the intact embryo? In those cases where most of the posterior ectoderm is removed (-2c2d), no shell forms in any part of the embryo. Yet in isolated half-, quarter-, or lobeless embryos, shell is secreted. Is there an integrative phenomenon which allows only one shell to form in an embryo? If there is, it can only occur when the polar lobe is present, for in its absence several internal shell masses may be formed. Only when the D quadrant (the polar lobe cytoplasm) is present do the other quadrants fail to form shell, even in those cases where in the absence of ectoderm the D quadrant will not. It then appears that the polar lobe exerts an inhibitory influence on the other quadrants which prevents differentiation leading to shell secretion.

The D quadrant has a complex role in shell formation. It gives rise to the $2 \mathrm{~d}$ micromere as the source of cells, it inhibits histogenesis leading to shell secretion in the $\mathrm{A}, \mathrm{B}$, and $\mathrm{C}$ quadrants, and it has a morphogenetic role, which not only affects the shell but also the form of the foot, velum, and eyes. Clement ('62) has shown, through his $\mathrm{D}$ quadrant macromere deletion studies, that external shell develops only after $2 \mathrm{~d}$ is formed. Even though all of the ectoderm used in the formation of the shell is present at this time, the shell is never morphologically normal. It is only after the formation of the third quartet, which contributes the $3 \mathrm{~d}$ micromere to form the left side of the foot, that a normal shell is formed. Since 3d does not contribute to the shell and since its removal has no effect on shell formation or morphogenesis, then there must be an organizing influence exerted by the 3D macromere on the overlying ectoderm. The presence of the $3 \mathrm{D}$ macromere during this period is then critical for morpohgenesis even though removal of its progeny $4 \mathrm{~d}$ and $4 \mathrm{D}$, two hours later, has no effect on morphogenesis of the shell. The ectoblast plus macromere combinations give added support to Clement's proposal for a temporally specific morphogenetic influence exerted at the third quartet stage. Removal of the 3D macromere and either two of the other three macromeres after the third quartet has formed results in an embryo with a small but essentially normal shell. The morphogenetic influence therefore has been exerted prior to removal of 3D. The other macromeres retain the capability to induce the histogenetic differentiation necessary for shell secretion by the ectoderm at some time between the first and sixth days of development. The mesentoblast and ectoblast combinations also give similar support. In all of these cases, the morphogenesis of the foot, velum, and eyes are also regulated just as Clement described from his $D$ macromere deletion studies.

Clement ('66) has further shown that the morphogenetic influence is not simply due to the nutritive value of the yolk since nucleated vegetal halves containing almost no yolk will develop into small but morphogenetically complete veligers.

The fact that an isolated yolk tag or polar lobe can induce shell material points to the uniqueness of the vegetal half and particularly that of the $\mathrm{D}$ quadrant. Costello ('45) has made clear the necessity for critical evaluation of the quality of the contact between the inducing and responding tissues in studying induction in mosaic development, especially in experimental systems such as those utilizing polar lobes. It appears that an actual fusion of the ectoblast cells to the polar lobe is required for shell to be formed. Whether or not the polar lobe membrane persists after this fusion has not been determined satisfactorily but the observations so far made suggest that it does persist. The $4 \mathrm{D}$ macro- 
mere does retain its membrane intact, at least in the region of the shell gland. The same kind of contact appears to be required for the various ectoderm-endoderm combinations for shell secretion. On the other hand, when the lobe is removed in calcium-free sea water, the lobe can induce the ectoblast to form shell after close contact. If there is any fusion in these cases, it is not strong enough to keep the ectoblast and lobe together during their removal from the depression in the operating dish. Since short treatment with calcium-free artificial sea water at this stage has no major effects on later development if the lobe is not separated, it appears that the cell membrane over the polar lobe serves as a significant barrier to the inductive process.

Since shell can be secreted for some time after the separation of the ectoderm from the endoderm, it appears that the inductive process is not simply a passage of molecules through the ectoderm to form shell in the overlying matrix as has been proposed by some investigators (Fretter and Graham, '62). This could not be the case in the early shell gland anyway because an elaborate series of differentiative events must precede the secretion of shell material.

This of course raises the question as to the nature of the interaction which can occur between cells and a nonnucleate region of cytoplasm where the plasma membrane of the latter may play a significant role. At present, one attractive possibility involves that portion of the yolk platelets which shows birefringence as first described by Clement ('62). These are distributed initially throughout the yolk but later appear to be predominantly localized in the D macromere. They disappear near the shell gland during shell synthesis and are completely lost from the endoderm by the time the larval shell is completed. The presence of alkaline phosphatase associated with some yolk granules gives support to this idea but one cannot be certain that these are the same granules as those which are birefringent. If the birefringent yolk granules did serve as a calcium reserve for shell secretion, the level of the substrate itself could be a major factor in the endoderm, causing the histogenetic differ- entiation which precedes the secretion of shell.

The paper by Davidson et al. ('65) presents a very attractive hypothesis for the role of the polar lobe as a differential activator of specific genes but the evidence presented to support the concept is yet inadequate. It is hoped that more evidence will be forthcoming and that it can bridge the gap between the time of known polar lobe interaction at the third quartet stage and the synthesis of RNA, some 24 hours later.

\section{Evolution of $D$ quadrant specialization}

Costello ('55) pointed out that spiral cleavage is characteristic of many more animal groups than was originally thought. Many such animals have undergone extensive modifications in their cleavage patterns but enough of the basic characteristics of spiral cleavage remain so that there is little doubt about the origin. The acoel flatworms were the first group to develop spiral cleavage. It appears, on the basis of meagre evidence, that the three duets of micromeres give rise to the ectoderm and some to the ectomesenchyme while the pair of macromeres and the fourth duet form the endoderm and inner mesoderm. (For a review, see Hyman, '51.)

Along the main evolutionary line of animals which have the basic spiralian plan are the polyclad flatworms which have developed the typical four quadrants, with the first three quartets forming the ectoderm. The macromeres and all of the fourth quartet cells except $4 \mathrm{~d}$ are nutritive. Some micromeres form ectomesenchyme but the mesoderm and all of the endoderm come from the left posterior micromere of the fourth quartet, the mesentoblast cell (4d). This is the first and most constant specialization of the $\mathrm{D}$ quadrant.

The nemertines have evidently experimented extensively with mesoderm formation, and mesoderm has been attributed to the micromeres, the $3 \mathrm{D}$ macromere, and the mesentoblast.

In annelids, the D quadrant also has additional significance in that the primary somatoblast (2d) and the mesentoblast are necessary for the formation of most of the adult worm and are held in reserve in the 
trochophore. The segmentation characteristic of the group requires the $\mathrm{D}$ quadrant material present in the mesoderm. The highly determined cleavage pattern appears to be of adaptive value because it allows the larva to form rapidly, with the least possible chance for error from cellular displacement due to the stress of the marine environment. Even here though an interaction is required between the ectoderm and endoderm for gastrulation to occur (Costello, '45).

In mollusks, the $\mathrm{D}$ quadrant becomes even more important and begins its influence early in development (Clement, '52, '56, '62), thereby controlling the symmetry and pattern of cleavage as well as the general form of the embryo by acting as the "morphogenetic organizer." The major modifications which transform the trochophore into the veliger, the foot, shell, velar lobe and paired eyes, are dependent on this organizer. The ontogeny of the veliger is then intertwined with that of the trochophore, in the early interactions of the polar lobe which result in apical tuft formation and also the third quartet stage morphogenetic interactions. Superimposed on the trochophore are such inductive processes as that between the ectoderm and endoderm which is required for histogenesis of shell, and there will undoubtedly be many others as our information becomes more complete.

The trend in $\mathrm{D}$ quadrant specialization is known in at least two other groups, the cephalopods and the barnacles. In the former, the $A, B$, and $C$ quadrants have been lost or reduced beyond recognition. The bilateral divisions of the micromeres of the remaining $D$ quadrant, which occur only in the later stages in the yolky eggs of some gastropods, are present from the first in the cephalopods. In the barnacles, the $A, B$, and $C$ quadrants have been completely lost but the cleavage of the remainder is typical spiral cleavage of the D quadrant.

The modifications and specializations in the D quadrant of spiralians evidently represent a very old and plastic system which has played a major role in the evolution of many of the invertebrate phyla.

\section{ACKNOWLEDGMENTS}

I wish to thank Professor A. C. Clement for his encouragement during the course of this investigation.

This work was supported by grants from the University of Michigan Cancer Research Committee and the National Science Foundation (GB-1035).

\section{LITERATURE CITED}

Cather, J. N. 1958 Fixing and staining the eggs of invertebrates. Stain Tech., 33: 146-147. 1963 A time schedule of the meiotic and early mitotic stages of Ilyanassa. Caryologia, 16: 663-670.

1966 Induction of the shell gland by transplanted polar lobes in Ilyanassa. Biol. Bull., 131: 386.

Clement, A. C. 1952 Experimental studies on germinal localization in Ilyanassa. I. The role of the polar lobe in determination of the cleavage pattern and its influence in later development. J. Exp. Zool., 121: 593-626.

1956 Experimental studies on germinal localization in Ilyanassa. II. The development of isolated blastomeres. J. Exp. Zool., 132: $427-446$.

- 1958 Discussion of paper by F. E. Lehmann in The Chemical Basis of Development. W. D. McEIroy and B. Glass, eds. Johns Hopkins Press, Baltimore, pp. 92-93.

- 1960 Development of the Ilyanassa embryo after removal of the mesentoblast cell. Biol. Bull., 119: 310 .

1962 Development of Ilyanassa following removal of the $\mathrm{D}$ macromere at successive cleavage stages. J. Exp. Zool., 149: 193-216.

1966 Cleavage and differentiation of the vegetal half of the Ilyanassa egg after removal of most of the yolk by centrifugal force. Biol. Bull., 131: 387.

Clement, A. C., and J. N. Cather 1957 A technique for preparing whole mounts of veliger larvae. Biol. Bull., 113: 340.

Collier, J. R., and M. McCann-Collier 1964 Shell gland formation in the Ilyanassa embryo. Exp. Cell Res., 34: 512-514.

Conklin, E. G. 1897 The embryology of Crepidula. J. Morph., 13: 1-226.

Costello, D. P. 1945 Experimental studies of germinal localization in Nereis. $I$. The development of isolated blastomeres. J. Exp. Zool, 100: 19-66.

1955 Cleavage, blastulation and gastrulation. In: Analysis of Development. B. H. Willier, P. A. Weiss and V. Hamburger, eds. W. B. Saunders, Philadelphia, chap. 2, pp. 213229.

Crampton, H. E. 1896 Experimental studies on gasteropod development. Roux' Arch. Entw.mech., 3: 1-19.

Davidson, E. H., G. W. Haslett, R. J. Finney, V. G. Alfrey and A. E. Mirsky 1965 Evidence for prelocalization of cytoplasmic factors affecting gene activation in early embryogenesis. Proc. Nat. Acad. Sci., 54: 696-703. 
Fretter, V., and A. Graham 1962 British Prosobranch Molluses. The Ray Society, London.

Hess, O. 1956a Die Entwicklung von Halbkeimen bei dem Süsswasser-Prosobranchier Bithynia tentaculata. Roux' Arch. Entw.-mech, 148: 336-361.

1956b Die Entwicklung von Exogastrula-Keimen bei dem Süsswasser-Prosobranchier Bithynia tentaculata. Roux' Arch. Entw.mech., 148: 474-488.

1957 Die Entwicklung von Halbkeimen bei dem Süsswasser-Pulmonaten Limnaea stagnalis. Roux' Arch. Entw.-mech., 150: 124-145. 1962 Entwickslungsphysiologie der Mollusken. Fort. d. Zool., 14: 130-163.

Hörstadius, S. 1937 Experiments on determination in the early development of Cerebratulus. Biol. Bull., 73: 317-342.

Hyman, L. H. 1951 The Invertebrates. vol. II. McGraw-Hill, New York.

Lillie, F. R. 1895 The embryology of the Unionidae. J. Morph., 8: 569-578.

Manigault, $P$. 1960 Coquille des mollusques: structure et formation. Traité de Zoologie, Tome V, fas. II, pp. 1823-1844. Masson et Cie, Paris.

Morrill, J. B., and D. M. Gottesman 1960 Development of isolated blastomeres of Limnaea palustris. Anat. Rec., 137: 383.

Novikoff, A. B. 1938a Embryonic determination in the annelid, Sabellaria vulgaris. 1 . The differentiation of ectoderm and endoderm when separated through induced exogastrulation. Biol. Bull., 74: 198-210.

$1938 b$ Embryonic determination in the annelid, Sabellaria vulgaris. II. Transplantation of polar lobes and blastomeres as a test of their inducing capacities. Biol. Bull., 74: 211-234.

Pearse, A. G. E. 1961 Histochemistry, Theoretical and Applied. Second Edition. Little, Brown and Co., Boston.

Penners, A. 1937 Regulation am Keim von Tubifex rivulorum Lam. nach Ausschaltung des ektodermalen Keimstreifs. Zeit. f. wiss. Zool., Bd. 149, S. 86-130.

Ranzi, S. 1931 Sviluppo di parti isolate di embrioni di Cefalopodi (Analisi sperimentale dell' embriogenesi). Pub. Staz. Zool. Napoli, 11: 104-146.

Raven, C. P. 1952 Morphogenesis in Limnaea stagnalis and its disturbance by lithium. J. Exp. Zool., 121: 1-77.

1958 Morphogenesis: The Analysis of Molluscan Development. Pergamon Press, New York.

Verdonk, N. H. 1965 Morphogenesis of the head region in Limnaea stagnalis L. Proeschrift. Rijkuniversiteit Te Utrecht, 1-133.

Wilbur, K. M. 1964 Shell formation and regeneration. In: Physiology of Mollusca. K. M. Wilbur and C. M. Yonge, eds. Academic Press, New York. Chap. 8, pp. 243-282.

Wilson, E. B. 1904a Experimental studies on germinal localization. 1 . The germ regions in the egg of Dentalium. J. Exp. Zool., 1: 1-72.

$1904 \mathrm{~b}$ Experimental studies on germinal localization. II. Experiments on the cleavage-mosaic in Patella and Dentalium. J. Exp. Zool., 1: 197-268. 